\title{
MicroRNA-206 regulates the epithelial-mesenchymal transition and inhibits the invasion and metastasis of prostate cancer cells by targeting Annexin A2
}

\author{
NING YANG ${ }^{1}$, LING WANG $^{2}$, JUN LIU $^{1}$, LI LIU $^{1}$, JIANGBO HUANG ${ }^{1}$, XIAN CHEN ${ }^{1}$ and ZHIGANG LUO ${ }^{1}$ \\ ${ }^{1}$ Department of Urology, The Second Affiliated Hospital of University of South China; \\ ${ }^{2}$ Department of Pharmacology, The Medical School of Hunan University of Environment and Biology, \\ Hengyang, Hunan 421001, P.R. China
}

Received November 20, 2016; Accepted February 19, 2018

DOI: $10.3892 /$ ol.2018.8395

\begin{abstract}
The present study investigated the molecular mechanism by which microRNA-206 (miR-206) targets Annexin A2 (ANXA2) expression and inhibits the invasion and metastasis of prostatic cancer cells through regulation of the epithelial-mesenchymal transition (EMT). Using bioinformatics analysis, miR-206 was identified as the most promising candidate miRNA that targeted ANXA2. Prostate tissue specimens from 60 patients with prostate cancer, 30 patients with metastatic prostate cancer and 20 patients with benign prostatic hyperplasia (BPH) were examined for ANXA2 protein expression by immunohistochemistry and western blotting and for miR-206 expression by reverse transcription-quantitative polymerase chain reaction. Additionally, human prostate cancer PC-3 cells were transfected with miR-206 mimics, miR-206 inhibitors or a negative control sequence, and expression of ANXA2, E-cadherin and N-cadherin was detected by western blotting. Transwell assays were performed to determine the effect of altered miR-206 expression on the invasive behavior of PC-3 cells. Bioinformatics analysis predicted complementary binding between miR-206 and ANXA2 mRNA. ANXA2 protein expression was detected in a significantly higher proportion of BPH tissues $(95 \%, 19 / 20)$ when compared with prostate cancer tissues $(51.7 \%, 31 / 60 ; \mathrm{P}<0.05)$. Similarly, ANXA2 was expressed in a significantly higher proportion of metastatic prostate cancer samples than that of prostate cancer samples $(\mathrm{P}<0.05)$. Expression of miR-206 was higher than that of ANXA2 in prostate cancer samples, but lower in BPH samples. Inhibition of miR-206 expression in PC-3 cells upregulated ANXA2 and E-cadherin protein
\end{abstract}

Correspondence to: Professor Zhigang Luo, Department of Urology, The Second Affiliated Hospital of University of South China, 35 Jiefang Road, Hengyang, Hunan 421001, P.R. China E-mail:1zg_nhdx@126.com

Key words: microRNA, prostate cancer, invasion, metastasis, Annexin A2, epithelial-mesenchymal transition expression levels, downregulated $\mathrm{N}$-cadherin and vimentin, and promoted cell invasion in vitro. These data suggested that binding between miRNA-206 and ANXA2 mRNA may regulate EMT signaling, thereby suppressing the invasion and metastasis of prostatic cancer cells.

\section{Introduction}

The growth rate of prostate cancer is slow and early symptoms are often not obvious and are easily overlooked, which may delay diagnosis and initiation of treatment $(1,2)$. The molecular mechanisms underlying prostate cancer metastasis remain unclear, and there is a requirement for the identification of molecular markers for metastasis to enable the treatment and to improve the prognosis of patients with prostate cancer.

The membrane-associated Annexin family is a group of highly evolutionarily conserved calcium-dependent phospholipid-binding proteins (3). The Annexin family has numerous critical functions in cell signaling, inflammation, cell proliferation and differentiation, and maintenance of the extracellular matrix integrity $(4,5)$. Downregulation or dysfunction of Annexins may serve important roles in the development and progression of malignant tumors. Annexin A2 (ANXA2) is one of nearly 20 members of the Annexin superfamily and contains a $\sim 70$ amino acid conserved repeat region (6). ANXA2 is involved in diverse cellular functions, including cell apoptosis, membrane transport, signal transduction and cell-cell interaction (7). ANXA2 is a multifunctional protein that serves an important regulatory role in biofilm formation, endocytosis and exocytosis, osteoblast formation, bone resorption, DNA synthesis, and cell proliferation and differentiation (8). ANXA2 is also associated with a number of cellular activities by connecting membrane protein complexes with the actin cytoskeleton, and is involved in ion channel formation, plasminogen activation and cellular matrix interactions $(9,10)$.

Epithelial-mesenchymal transition (EMT) is a highly regulated process by which epithelial cells transform into a mesenchymal cell phenotype (11). EMT is associated with primary tumor invasion, cell migration and secondary 
metastasis formation in a variety of tumor types, particularly in those of epithelial origin $(12,13)$.

Bioinformatics analysis is a major tool for the identification of putative microRNA (miRNA/miR)-targeted genes. The present study used bioinformatics software analysis to identify ANXA2 mRNA as a target of miR-206 and an aim of the present study was to examine the molecular mechanism by which miR-206 targeting of $A N X A 2$ regulates the EMT, and the invasive and metastatic activity of prostate cancer cells.

\section{Materials and methods}

Patients and tissue specimens. A total of 110 male patients were enrolled in the present study; 60 with prostate cancer (median age, 72.8 years; age range, 56-85 years), 30 with metastatic prostate cancer (median age, 73.5 years; age range, $57-85$ years), and 20 with benign prostatic hyperplasia ( $\mathrm{BPH}$; median age, 68.6 years; age range, 52-83 years) as control. Patients with prostate cancer included in the present study received no preoperative medication and had no history of surgical castration or radiotherapy. Patients with BPH had no long-term medication history prior to surgery. Tissue samples were obtained by surgical resection at the Department of Urology at the Second Affiliated Hospital, University of South China (Hengyang, China) and were stored at $-80^{\circ} \mathrm{C}$ prior to use. All specimens were reviewed independently by two senior pathologists and the diagnoses were confirmed by histopathological examination. The present study was certified by the Ethics Committee of the Second Affiliated Hospital of University of South China, and all participants provided written informed consent.

Cell lines. The prostate cancer PC-3 cell line was purchased from The Cell Centre of Central South University (Changsha, China). Cells were cultured in RPMI-1640 medium (Gibco; Thermo Fisher Scientific, Inc., Waltham, MA, USA), supplemented with $10 \%$ bovine serum albumin, and were incubated at $37^{\circ} \mathrm{C}$ in a $5 \% \mathrm{CO}_{2}$ atmosphere.

Reagents. The immunohistochemical streptavidin peroxidase (S-P) kit and 3,3'-diaminobenzidine developer were obtained from Fuzhou Maixin Biotech Co., Ltd. (Fuzhou, Fujian province, China). Mouse anti-human monoclonal antibodies against ANXA2, GAPDH, E-cadherin, $N$-cadherin and $\beta$-actin were purchased from Santa Cruz Biotechnology, Inc. (Dallas, TX, USA). Lipofectamine 2000 was purchased from Invitrogen; Thermo Fisher Scientific, Inc., the Transwell assay kit was purchased from Corning Incorporated (Corning, NY, USA), and Matrigel was obtained from BD Biosciences (Franklin Lakes, NJ, USA).

Bioinformaticsanalysis.miRNAs predicted to bind to ANXA2 mRNA were identified using the miRWalk online program, which contains 10 software programs (http://zmf.umm.uniheidelberg.de/apps/zmf/mirwalk/predictedmirnagene.html). The miRNAs with the highest predicted binding scores were identified using miRanda software (version: August 2010 release; http://www.microrna.org/microrna/home.do), which computes thermodynamic stability scores and sequence conservation scores.
Immunohistochemistry (IHC). The prostate tissue specimens were fixed using by $10 \%$ formalin for $24-48 \mathrm{~h}$ at room temperature, and then embedded in paraffin. The sample was sliced into sections $4 \mu \mathrm{m}$ thick. Immunohistochemical staining of prostate tissue specimens was performed using the S-P immunohistochemical method (14). The cytoplasmic staining intensity was scored by two pathologists as follows: No color, negative (-); pale yellow, weakly positive $(+)$; brown, positive $(++)$; and tan, strongly positive $(+++)$. The percentage of tissue samples with positive expression was calculated as [(total number of samples with weakly positive + positive + strongly positive staining)/total number of samples evaluated] x100.

RNA extraction. Total RNA was extracted from fresh prostate cancer and $\mathrm{BPH}$ tissues by homogenization using TRIzol reagent (Thermo Fisher Scientific, Inc. Waltham, MA, USA). Following incubation for $5 \mathrm{~min}$ at room temperature, the samples were mixed with $200 \mathrm{ml}$ of chloroform, incubated for $5 \mathrm{~min}$ at room temperature, and then centrifuged at $12,000 \mathrm{x} \mathrm{g}$ for $15 \mathrm{~min}$ at $4^{\circ} \mathrm{C}$. The supernatant was removed, combined with $200 \mathrm{ml}$ isopropanol, mixed by inversion, incubated for $10 \mathrm{~min}$ at room temperature, and centrifuged at $12,000 \mathrm{x} \mathrm{g}$ for $15 \mathrm{~min}$ at $4^{\circ} \mathrm{C}$. The supernatant was removed and the pellet was washed by addition of $1 \mathrm{ml}$ ethanol followed by centrifugation at $12,000 \mathrm{x} \mathrm{g}$ for $15 \mathrm{~min}$ at $4^{\circ} \mathrm{C}$. The supernatant was removed and the pellet was vacuum dried. Finally, the RNA was resuspended in deionized water and subsequently used for reverse transcription-quantitative polymerase chain reactions (RT-qPCR).

$R T-q P C R$. PCR amplification of miR-206 was performed using a two-step method using an RT-qPCR kit (Sigma-Aldrich; Merck KGaA, Darmstadt, Germany). RT were performed in a water bath at $50^{\circ} \mathrm{C}$ for $60 \mathrm{~min}$ and $15 \mathrm{~min}$ with inactivated reverse transcriptase (Sigma-Aldrich; Merck KGaA, Darmstadt, Germany) at $70^{\circ} \mathrm{C}$ in a water bath. Primers were designed based on the precursor sequence of miR-206 by Primer Premier 5.0 (Primer Biosoft, Paolo Alto, CA, USA). Primer sequences were as follows: miR-206 forward, 5'-TGC TTCCCGAGGCCACATGC-3' and reverse, 5'-GTGTGTGGT TTCGGCAAGTG-3'; and U6 forward, 5'-GCTTCGGCA GCACATATACTAAAAT-3' and reverse, 5'-CGCTTCACG AATTTGCGTGTCAT-3'. All primers were synthesized by Guangzhou RiboBio Co., Ltd. (Guangzhou, China). The reactions were performed on a fluorescence RT-qPCR instrument, according to the manufacturer's protocol. The PCR reaction system is as follows: $2.5 \mu \mathrm{ldNTP}(2.5 \mathrm{mM}$ each); $2.5 \mu 110 \mathrm{x}$ PCR buffer; $1.5 \mu 1 \mathrm{MgCl}_{2}$ solution; $1 \mathrm{U}$ Taq polymerase; $0.25 \mathrm{x}$ SYBRGreen I (Sigma-Aldrich; Merck KGaA) (final concentration); $1 \mu \mathrm{l} 10 \mu \mathrm{M}$ PCR forward and reverse primer; $1 \mu \mathrm{l} \mathrm{cDNA}$; water (to a total volume of $25 \mu \mathrm{l}$ ). The $\mathrm{U} 6$ reaction was as follows: $95^{\circ} \mathrm{C}$ for $5 \mathrm{~min}$ and 35 cycles of $95^{\circ} \mathrm{C}$ for $10 \mathrm{sec}, 59^{\circ} \mathrm{C}$ for $15 \mathrm{sec}, 72^{\circ} \mathrm{C}$ for $20 \mathrm{sec}$ and $82^{\circ} \mathrm{C}$ for $5 \mathrm{sec}$. The miRNA reaction was as follows: $95^{\circ} \mathrm{C}$ for $15 \mathrm{~min}$ and 40 cycles of $94^{\circ} \mathrm{C}$ for $15 \mathrm{sec}, 55^{\circ} \mathrm{C}$ for $30 \mathrm{sec}$ and $70^{\circ} \mathrm{C}$ for $30 \mathrm{sec}$ ). The $2^{-\Delta \Delta \mathrm{Cq}}$ was used to quantify the results (15).

Western blot analysis. To extract total cellular protein, tissues or cells were mixed with precooled lysis buffer (Sigma-Aldrich; Merck KGaA), centrifuged for $10 \mathrm{~min}$ at $4^{\circ} \mathrm{C}$ at $10,000 \mathrm{x} \mathrm{g}$, and placed on ice for $30 \mathrm{~min}$. Following 

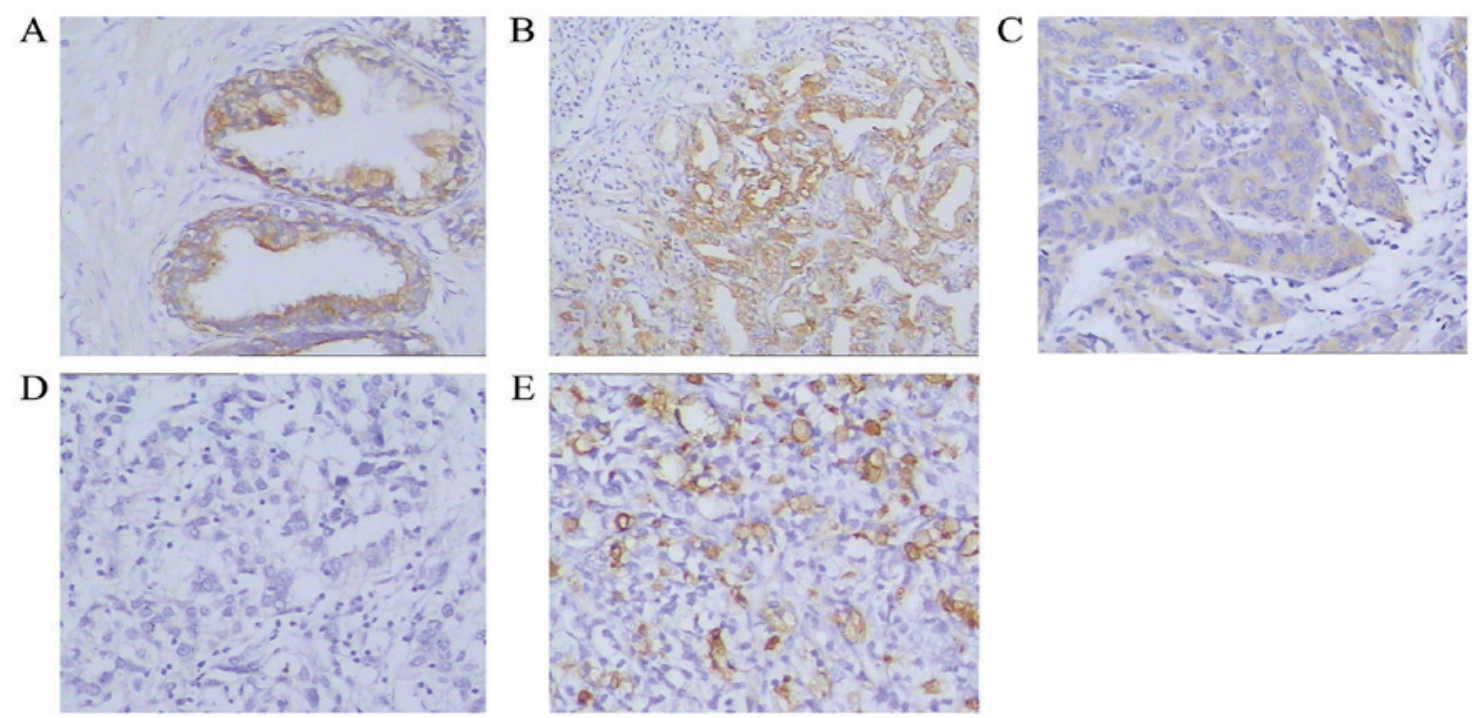

Figure 1. Expression of ANXA2 in prostate cancer and BPH tissues via immunohistochemical staining. (A) BPH (++), (B) highly differentiated prostate cancer $(+++),(C)$ moderately differentiated prostate cancer (+), (D) poorly differentiated prostate cancer (+/-)and (E) metastatic prostate cancer (++). Magnification, $\mathrm{x} 200$. ANXA2, Annexin A2; BPH, benign prostate hyperplasia.

centrifugation, the supernatants were removed and protein concentrations were determined using a Bradford assay. A total of $40 \mu \mathrm{g}$ protein was separated using SDS-PAGE $(10 \%$ gel). The proteins were denatured by incubation for $5 \mathrm{~min}$ at $100^{\circ} \mathrm{C}$, separated by gel electrophoresis and transferred onto polyvinylidene difluoride membranes. The membranes were blocked at room temperature with in 5\% skimmed milk powder for 1-2 $\mathrm{h}$ and were then washed and incubated with the following primary antibodies: Anti-ANXA2 (1:1,000; cat. no. 28385), anti- $\beta$-actin (1:10,000; cat. no. 58673), anti-vimentin $(1: 1,500$; cat. no. 6260), anti N-cadherin (1:1,500; cat. no. 8424) and anti-E-cadherin (1:1,500; cat. no. 8426), overnight at $4^{\circ} \mathrm{C}$, and added the secondary antibody (1:1,000; HRP-conjugated mouse anti-rabbit IgG; cat. no. 2357 ) for $2 \mathrm{~h}$ at temperature. All the primary antibodies and the secondary antibody were purchased from Santa Cruz Biotechnology, Inc. (Dallas, TX, USA). The proteins were treated using enhanced chemiluminescence chromogenic solution (Boster Biological Engineering Co., Ltd. Wuhan, China). Visualization of the protein on the membrane occurred upon exposure to X-ray film. The Gray value was detected by Image $\mathbf{J}$ software (version K1.45; National Institutes of Health, Bethesda, MD, USA).

In vitro cell invasion assay. All the cell lines were cultured in RPMI-1640 and 10\% fetal bovine serum (Hangzhou Sijiqing Biological Engineering Co., Ltd., Hangzhou, China). PC-3 cells were seeded onto 6 -well plates $\left(\sim 2 \times 10^{5}\right.$ cells/plate $)$ and cultured to $80 \%$ confluence prior to transfection at $37^{\circ} \mathrm{C}$. Cells were transfected with miR-206 mimic or inhibitor sequences or a negative control (NC) sequence using a Lipofectamine 2000 transfection kit (Santa Cruz Biotechnology, Inc.). After $48 \mathrm{~h}$ of transfection, the cell extractive was analyzed to detect the activity of the reported gene. For the Transwell invasion assay, Matrigel was diluted at 1:100 in serum-free medium and $100 \mu \mathrm{l}$ was added to coat the upper chamber at room temperature for $24 \mathrm{~h}$. To detect invasion, the upper chamber was removed, and the cells in the membrane were fixed in ethanol at room temperature for $30 \mathrm{~min}$ and stained with $0.5 \%$ crystal violet at room temperature for $20 \mathrm{~min}$. The number of invaded tumor cells in the membrane was counted under an optical microscope (Nikon Corporation, Tokyo, Japan) at x400 magnification.

Statistical analysis. Data were analyzed using SPSS version 17 software (SPSS, Inc., Chicago, IL, USA). Data are expressed as the mean \pm standard deviation. Comparisons were performed using an unpaired Student's t-test or Mann-Whitney U test for two groups or with a one-way analysis of variance, followed by the least significant difference post hoc test, for three groups. $\mathrm{P}<0.05$ was considered to indicate a statistically significant difference.

\section{Results}

miRNA-ANXA2 interactions predicted by miRWalk. Using miRWalk, which combines the power of 10 programs, the following 12 miRNAs predicted to interact with ANXA2 mRNA were identified: hsa-miR-206, hsa-miR-1, hsa-miR-9, hsa-miR-613, hsa-miR-185, hsa-miR-425, hsa-miR-890, hsa-miR-155, hsa-miR-20b, hsa-miR-520h, hsa-miR-579 and hsa-miR-767-5p (Table I).

Optimal miRNA-ANXA2 interactions predicted by miRanda. miRNAs with the highest binding scores for ANXA2 mRNA were identified using miRanda software, which incorporates thermodynamic and sequence conservation scores. This analysis identified the following 4 miRNAs that met the imposed criteria (mirSVR, $\leq-0.1$; phastCons, 0.5-0.7): miR-206, miR-1, miR-613 and miR-425. miR-206 was predicted to bind to ANXA2 mRNA with the highest stability and specificity, suggesting that it may exhibit preferential binding to ANXA2 mRNA (Table II). 


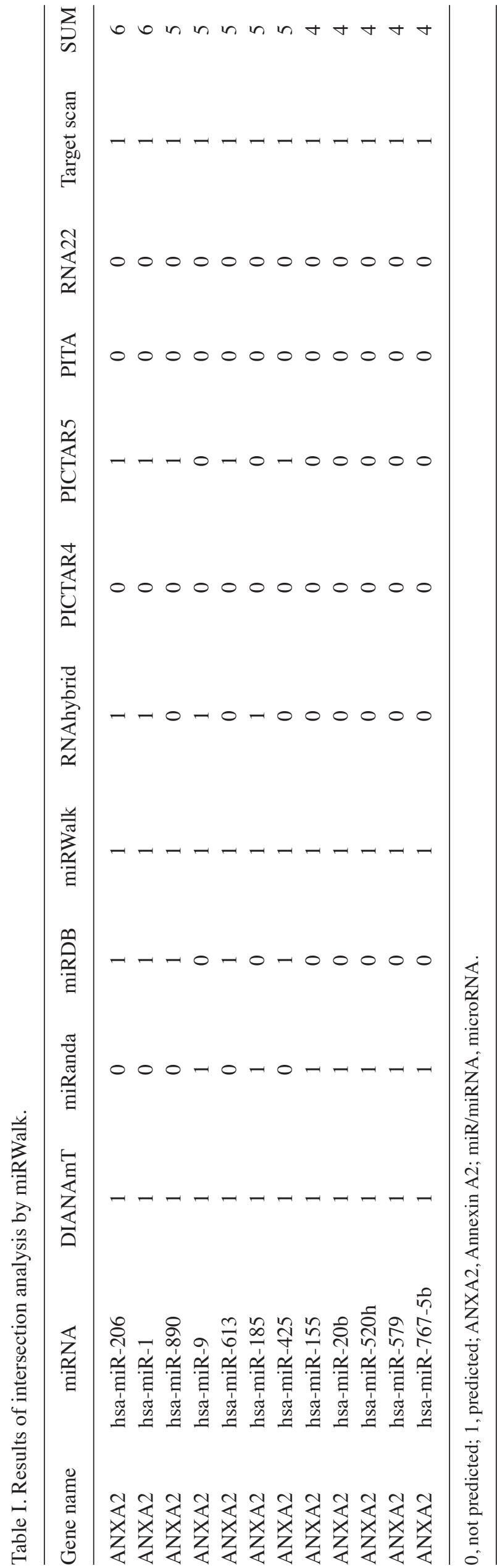

Table II. Scores of mirSVR and PhastCons among 12 predicted miRNAs.

mirSVR score Ranking PhastCons score

\begin{tabular}{llll}
\hline hsa-miR-206 & -1.1965 & 1 & 0.6944 \\
hsa-miR-1 & -1.1964 & 2 & 0.6944 \\
hsa-miR-613 & -1.1865 & 3 & 0.6944 \\
hsa-miR-425 & -1.1787 & 4 & 0.6451 \\
hsa-miR-520h & -0.8678 & 5 & 0.6697 \\
hsa-miR-185 & -0.7357 & 6 & 0.6459 \\
hsa-miR-579 & -0.4511 & 7 & 0.5177 \\
hsa-miR-9 & -0.2252 & 8 & 0.6459 \\
hsa-miR-155 & -0.2098 & 9 & 0.5084 \\
hsa-miR-767 & -0.1488 & 10 & 0.5022 \\
hsa-miR-890 & -0.1193 & 11 & 0.6944 \\
hsa-miR-20b & -0.0235 & 12 & 0.6697 \\
\hline
\end{tabular}

miR/miRNA, microRNA.

Expression of ANXA2 protein in prostate cancer and $B P H$ tissues. Expression of ANXA2 protein in tissue specimens was assessed by IHC. The proportion of samples positively stained for ANXA2 was significantly higher in the BPH samples $(95 \%, 19 / 20)$ compared with the prostate cancer samples $(51.7 \%, 31 / 60 ; \mathrm{P}<0.001)$. Additionally, $76.7 \%$ (23/30) of the metastatic prostate cancer tissues exhibited positive ANXA2 staining, which was a significantly higher proportion than that of the prostate cancer samples ( $\mathrm{P}<0.001$ Fig. 1; Table III).

Association between the expression of miR-206 and ANXA2 in prostate cancer and BPH tissues. The expression of miR-206 was significantly higher in prostate cancer tissues than in BPH tissues, while an inverse expression pattern was observed for ANXA2 (P<0.001; Fig. 2). miR-206 expression was significantly increased in the prostate cancer samples compared with the BPH samples $(\mathrm{P}<0.001)$. ANXA2 expression levels were significantly decreased in the prostate cancer samples, but increased in the BPH samples $(\mathrm{P}<0.001)$. By contrast, miR-206 was lowly expressed and ANXA2 was highly expressed in the BPH samples. Therefore, there was a negative association between the expression of miR-206 and ANXA2.

In vitro invasion assay of a prostate cancer cell line. The invasion assay demonstrated that a significantly lower number of PC-3-miR-206-inhibitor cells exhibited invasive behavior than untransfected PC-3 or PC-3-miR-206-NC cells (Fig. 3; $\mathrm{P}<0.001$, data not shown). By contrast, the number of invasive PC-3-miR-206-mimic cells was significantly higher than the number of invasive PC-3 or PC-3-miR-206-NC cells. These results indicated that downregulation of miR-206 may promote the invasive capacity of PC-3 prostate cancer cells in vitro (Table IV; Fig. 3; $\mathrm{P}<0.001$ ).

Association between the expression of miR-206 and that of ANXA2 and WNT pathway proteins. Downregulation of miR-206 in PC-3-miR-206-inhibitor cells was associated 
Table III. Expression of ANXA2 in prostate cancer and BPH tissues.

Immunohistochemical staining scores

\begin{tabular}{|c|c|c|c|c|c|}
\hline & \multirow[b]{2}{*}{$\mathrm{n}$} & \\
\hline & & Weak positive or negative $(0-2)$ & Positive (3-4) & Strong positive (5-6) & P-value \\
\hline $\mathrm{BPH}$ & 20 & 1 & 3 & 16 & $<0.001^{\mathrm{a}}$ \\
\hline Prostate cancer & & & & & $<0.001^{\mathrm{b}}$ \\
\hline Highly-differentiated & 20 & 6 & 12 & 2 & \\
\hline Moderately-differentiated & 34 & 18 & 16 & 0 & \\
\hline Poorly-differentiated & 6 & 5 & 1 & 0 & \\
\hline Metastatic prostate cancer & 30 & 7 & 8 & 15 & $<0.001^{\mathrm{c}}$ \\
\hline
\end{tabular}

ANXA2, Annexin A2; BPH, benign prostatic hyperplasia. ${ }^{\mathrm{P}}<0.001$, BPH vs. prostate cancer; ${ }^{\mathrm{b}} \mathrm{P}<0.001$, among the three groups; ${ }^{\mathrm{C}}<0.05$ Metastatic prostate cancer vs. prostate cancer. Data were analyzed using Mann-Whitney U test or one-way analysis of variance followed by the least significant difference post hoc test.

Table IV. Changes in the effect of miR-206 expression on the invasion of prostate cancer cells in vitro.

\begin{tabular}{lcc}
\hline Group & no. invaded cells (/HPF) & P-value \\
\hline PC-3-miR-206-inhibitors & $107 \pm 16^{\mathrm{a}}$ & $<0.001$ \\
PC-3 & $52 \pm 9$ & \\
PC-3-miR-206-NC & $48 \pm 8$ & \\
PC-3-miR-206-mimics & $21 \pm 4^{\mathrm{b}}$ & $<0.001$ \\
\hline
\end{tabular}

${ }^{\mathrm{a}} \mathrm{P}<0.001 \quad \mathrm{PC}-3$-miR-206-inhibitors $\quad$ vs. $\mathrm{PC}-3 / \mathrm{NC} ; \quad{ }^{\mathrm{b}} \mathrm{P}<0.05$ PC-3-miR-206-mimics vs. PC-3/NC. miR, microRNA; NC, negative control; HPF, high power field. One-way analysis of variance followed by the least significant difference post hoc test.

with increased expression of ANXA2 and E-cadherin and decreased expression of N-cadherin and vimentin (Fig. 4). By contrast, upregulation of miR-206 in PC-3-miR-206-mimic cells resulted in downregulation of ANXA2 and E-cadherin and upregulation of $\mathrm{N}$-cadherin and vimentin ( $\mathrm{P}<0.05$; Fig. 4). PC-3 and PC-3-miR-206-NC cells exhibited no significant differences in the expression of ANXA2, E-cadherin, N-cadherin or vimentin (Fig. 4).

The results indicated that transfection of miR-206 mimics into PC-3 cells inhibited the expression of ANXA2, resulting in the downregulation of E-cadherin and vimentin and the upregulation of N-cadherin. However, transfection of miR-206 inhibitors into PC-3 cells upregulated ANXA2 expression and concomitantly downregulated E-cadherin. Therefore, the miR-206 target $A N X A 2$ regulates the expression of E-cadherin, $\mathrm{N}$-cadherin and vimentin and may be involved in the EMT.

\section{Discussion}

Changes in the expression and distribution of ANXA2 have been detected in many types of cancer, such as laryngeal cancer, pancreatic cancer, breast cancer, and so on (15-17). ANXA2 expression has also been associated with the differentiation of squamous cell lung cancer (18). ANXA2 content was demonstrated to be high in human hepatocellular cancer cells but not in embryonic tissues, normal liver cells or injured liver cells, suggesting that ANXA2 may serve a key role in hepatocellular carcinoma (19). A study undertaken by Kirshner et al (20) revealed that respiratory cilia, pleural mesothelial and alveolar epithelial cells exhibit a high expression of ANXA2. Expression of ANXA2 in prostate cancer differs from that in other tumor types. Immunohistochemical studies have demonstrated that ANXA2 highly expressed in $>50 \%$ of glands in the majority (>85\%) of patients with BPH (21). A number of studies have demonstrated that changes in the expression and distribution of ANXA2 are associated with the invasion and metastasis of various tumor types $(22,23)$. However, the exact molecular mechanism by which this occurs remains unclear.

In the present study, a significantly higher proportion of BPH samples than prostate cancer samples were positively stained for ANXA2 protein. Furthermore, a significantly higher proportion of metastatic cancer samples than prostate cancer samples expressed ANXA2. Therefore, the expression of ANXA2 may be a potential marker for the progression and development of prostate cancer.

The interaction between miRNAs and mRNAs occurs through complementary binding of base pairs. However, the interaction is not usually fully complementary, and several programs have been developed to predict miRNA-mRNA binding and to identify the specific target genes of miRNAs (24). Due to the fact that the bioinformatics programs use algorithms that focus on different aspects of the interactions between miRNAs and mRNAs, the prediction results are not entirely consistent between programs. Therefore, a previous study employed a combination of predictive programs for analyses (25). In the present study, ANXA2 mRNA was predicted by miRWalk online program, which contains 10 software programs), to be a target for 12 miRNAs, of which the interaction with miR-206 was predicted to have the highest stability and specificity. This suggested that miR-206 may preferentially target $A N X A 2$ mRNA.

The present study also revealed that the expression of miR-206 was higher than that of ANXA2 in prostate cancer samples, while an inverse association was observed in BPH tissues, suggesting a negative association between 
A

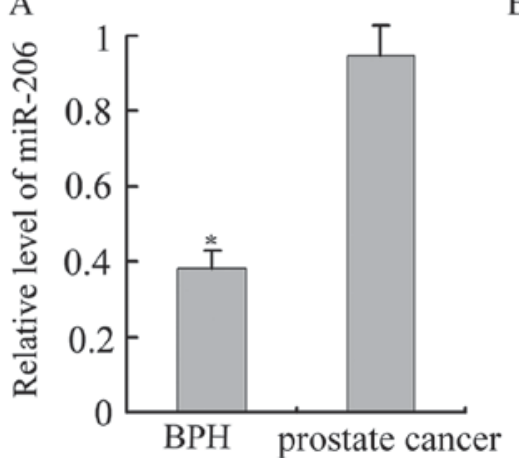

B

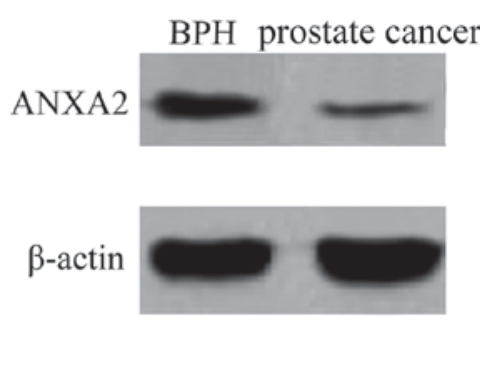

$\mathrm{C}$

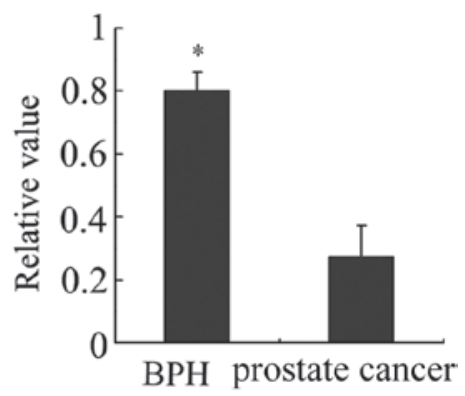

Figure 2. Association between the expression of miR-206 and ANXA2 in prostate cancer and BPH tissues. (A) Relative expression of miR-206 in prostate cancer and BPH tissues ("P<0.05). (B) Western blot analysis of ANXA2 expression in prostate cancer and BPH tissues. (C) Relative expression of ANXA2 based on western blotting results ("P<0.05). ANXA2, Annexin A2; BPH, benign prostate hyperplasia; miR, microRNA.
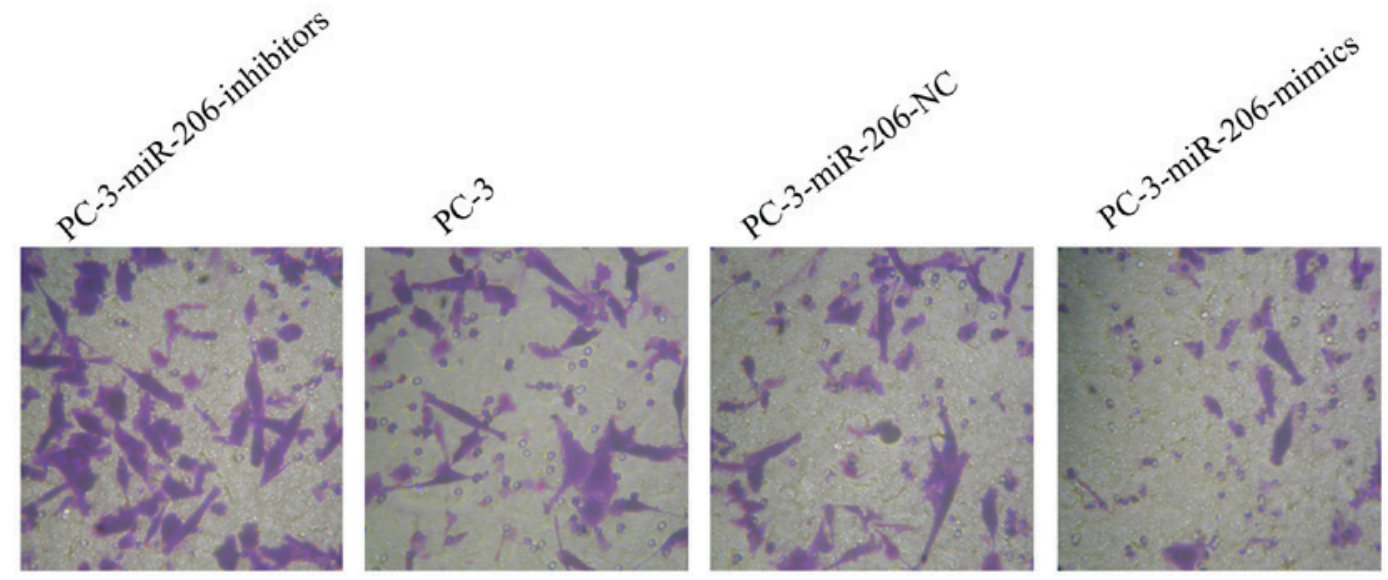

Figure 3. Invasion assay of prostate cancer cells, stained with crystal violet and visualized at x400 magnification. NC, negative control; miR, microRNA.
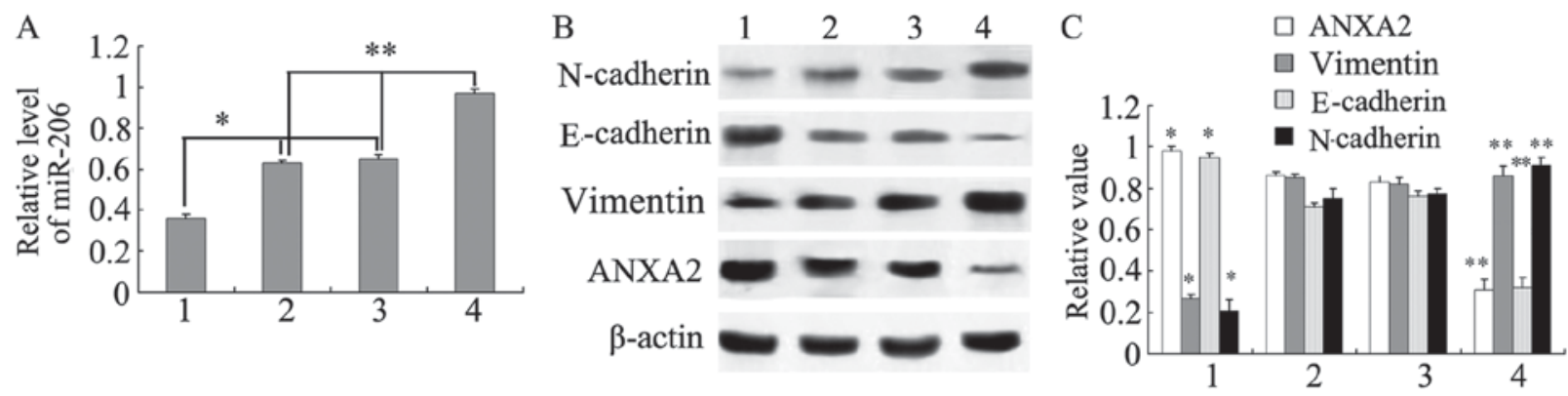

Figure 4. Association between the expression of miR-206, ANXA2, E-cadherin, N-cadherin and vimentin in prostate cancer cell lines. (A) Relative expression level of miR-206. (B) Western blot analysis of protein expression in 1) PC-3-miR-206-inhibitor, 2) PC-3, 3) PC-3-miR-206-NC and 4) PC-3-miR-206-mimic cells. (C) Statistical analysis of western blotting results. "P<0.05, PC-3-miR-206 inhibitor vs. PC-3 or PC-3-miR-206-NC; ${ }^{* *} \mathrm{P}<0.05$, PC-3-miR-206 mimic vs. PC-3 or PC-3-miR-206-NC. ANXA2, Annexin A2; NC, negative control; miR, microRNA; HPF, high power field.

the expression of miR-206 and ANXA2. These findings further supported the possibility that ANXA2 may be a target of miR-206.

Previous studies have demonstrated that miR-206 acts as a tumor suppressor, and that its expression is downregulated in many tumor types compared with normal cells or tissues (26). The expression of miR-206 is downregulated in renal cell carcinoma, and overexpression of miR-206 targets cell division kinase CDK9 and CDK4, inducing cell cycle arrest and inhibiting cell proliferation (26). miR-206 expression is also downregulated in colon cancer cells, and overexpression of miR-206 targets d-MET and inhibits cell proliferation and metastasis (27). miR-206 directly targets the oncogenes Kirsten rat sarcoma proto-oncogene and ANXA2, thereby acting as tumor suppressor in human pancreatic ductal adenocarcinoma cells by blocking cell cycle progression, and cell proliferation, migration and invasion (28). The miRNA functional analysis and a luciferase reporter assay demonstrated that miR-206 effectively downregulated the expression of ANXA2 by binding to the 3'-untranslated region of the ANXA2 mRNA 
in pulmonary artery smooth muscle cells (29). However, there have been no previous studies on miR-206 expression in prostate cancer.

EMT is a complex and dynamic process. One of the early events is remodeling of the cytoskeleton, which is accompanied by increased expression of mesenchymal markers, including $\mathrm{N}$-cadherin and vimentin, and decreased expression of epithelial markers, such as E-cadherin (30). A previous study demonstrated that low expression of E-cadherin may induce EMT (31). $\mathrm{N}$-cadherin is a cell surface adhesion molecule and a major structural element in intercellular adhesion. Its principal function is to mediate cell adhesion and migration (32). The expression of N-cadherin is increased in epithelial malignant tumors and is associated with the EMT (33). Recent studies have demonstrated that $\mathrm{E}$-cadherin and $\mathrm{N}$-cadherin are aberrantly expressed in numerous tumor types and serve an important role in the occurrence, development, invasion and metastasis of tumors $(34,35)$.

In the present study, miR-206 was demonstrated to promote the invasive ability of prostate cancer cells in vitro. Increased expression of miR-206 inhibited the expression of ANXA2, resulting in downregulation of E-cadherin, and upregulation of N-cadherin and vimentin. By contrast, decreased expression of miR-206 increased the expression of ANXA2, resulting in upregulation of E-cadherin and downregulation of $\mathrm{N}$-cadherin and vimentin. These results indicated that miR-206 may bind to ANXA2 and inhibit its expression, which, in turn, regulates the EMT and inhibits the invasion and metastasis of prostate cancer cells.

\section{Acknowledgements}

Not applicable.

\section{Funding}

The Hunan Province Science Foundation (grant no. 2015JC3088).

\section{Availability of data and materials}

The datasets used and/or analyzed during the current study are available from the corresponding author on reasonable request.

\section{Author's contributions}

NY contributed to the conception and design of the work. LW, JL, LL, JH and XC acquired and analyzed the data for the work.ZLdesigned the work and corrected the manuscript. All authors read and approved the final manuscript.

\section{Ethics approval and consent to participate}

The present study was certified by the Ethics Committee of the Second Affiliated Hospital of University of South China, and all participants provided written informed consent.

\section{Consent for publication}

All participants provided written consent agreeing for publication of the present study.

\section{Competing interests}

The authors declare that they have no competing interests.

\section{References}

1. Nandana S and Chung LW: Prostate cancer progression and metastasis: Potential regulatory pathways for therapeutic targeting. Am J Clin Exp Urol 2: 92-101, 2014.

2. Simons JW: Prostate cancer immunotherapy: Beyond immunity to curability. Cancer Immunol Res 2: 1034-1043, 2014.

3. Monastyrskaya K: Functional association between regulatory RNAs and the annexins. Int J Mol Sci 19: E591, 2018.

4. Mussunoor S and Murray GI: The role of annexins in tumour development and progression. J Pathol 216: 131-140, 2008.

5. Mortimer JC, Laohavisit A, Macpherson N, Webb A, Brownlee C, Battey NH and Davies JM: Annexins: Multifunctional components of growth and adaptation. J Exp Bot 59: 533-544, 2008.

6. Zhang M, Chen D, Zhen Z, Ao J, Yuan X and Gao X: Annexin A2 positively regulates milk synthesis and proliferation of bovine mammary epithelial cells through the mTOR signaling pathway. J Cell Physiol 233: 2464-2475, 2018

7. Sun MY, Xing RH, Gao XJ, Yu X, He HM, Gao N, Shi HY, $\mathrm{Hu}$ YY, Wang QX, Xu JH and Hou YC: ANXA2 regulates the behavior of SGC-7901 cells. Asian Pac J Cancer Prev 14: 6007-6012, 2013

8. Jeon YR, Kim SY, Lee EJ, Kim YN, Noh DY, Park SY and Moon A: Identification of annexin II as a novel secretory biomarker for breast cancer. Proteomics 13: 3145-3156, 2013.

9. Lokman NA, Elder AS, Ween MP, Pyragius CE, Hoffmann P, Oehler MK and Ricciardelli C: Annexin A2 is regulated by ovarian cancer-peritoneal cell interactions and promotes metastasis. Oncotarget 4: 1199-1211, 2013.

10. Zhang HJ, Yao DF, Yao M, Huang H, Wang L, Yan MJ, Yan XD, Gu X, Wu W and Lu SL: Annexin A2 silencing inhibits invasion, migration, and tumorigenic potential of hepatoma cells. World $\mathrm{J}$ Gastroenterol 19: 3792-3801, 2013

11. Zare M, Bastami M, Solali S and Alivand MR: Aberrant miRNA promoter methylation and EMT-involving miRNAs in breast cancer metastasis: Diagnosis and therapeutic implications. J Cell Physiol 233: 3729-3744, 2018.

12. Feng J, Fu Z, Guo J, Lu W, Wen K, Chen W, Wang H, Wei J and Zhang S: Overexpression of peroxiredoxin 2 inhibits TGF- $\beta 1$-induced epithelial-mesenchymal transition and cell migration in colorectal cancer. Mol Med Rep 10: 867-873, 2014.

13. Bezdenezhnykh N, Semesiuk N, Lykhova O, Zhylchuk V and Kudryavets Y: Impact of stromal cell components of tumor microenvironment on epithelial-mesenchymal transition in breast cancer cells. Exp Oncol 36: 72-78, 2014.

14. Mou H, Yu L, Zheng X, Liao Q, Hou X and Wu Y: p16 gene expression in pancreatic cancer tissue and its importance in diagnosis. J Biol Regul Homeost Agents 31: 1043-1047, 2017.

15. Luo S, Xie C, Wu P, He J, Tang Y, Xu J and Zhao S: Annexin $\mathrm{A} 2$ is an independent prognostic biomarker for evaluating the malignant progression of laryngeal cancer. Exp Ther Med 14: 6113-6118, 2017.

16. Murphy AG, Foley K, Rucki AA, Xia T, Jaffee EM, Huang CY and Zheng L: Stromal Annexin A2 expression is predictive of decreased survival in pancreatic cancer. Oncotarget 8: 106405-106414, 2017.

17. Shetty P, Patil VS, Mohan R, D'souza LC, Bargale A, Patil BR, Dinesh US, Haridas V and Kulkarni SP: Annexin A2 and its downstream IL-6 and HB-EGF as secretory biomarkers in the differential diagnosis of Her-2 negative breast cancer. Ann Clin Biochem 54: 463-471, 2017.

18. Cui JW and Wang YL: Expression and function of Annexin II in lung cancer tissue. Asian Pac J Trop Med 6: 150-152, 2013.

19. Dong Z, Yao M, Zhang H, Wang L, Huang H, Yan M, Wu W and Yao D: Inhibition of Annexin A2 gene transcription is a promising molecular target for hepatoma cell proliferation and metastasis. Oncol Lett 7: 28-34, 2014.

20. Kirshner J, Schumann D and Shively JE: CEACAM1, a cell-cell adhesion molecule, directly associates with annexin II in a three-dimensional model of mammary morphogenesis. J Bio Chem 278: 50338-50345, 2003. 
21. Yee DS, Narula N, Ramzy I, Boker J, Ahlering TE, Skarecky DW and Ornstein DK: Reduced annexin II protein expression in high-grade prostatic intraepithelial neoplasia and prostate cancer. Arch Pathol Lab Med 131: 902-908, 2007.

22. Ohno Y, Izumi M, Kawamura T, Nishimura T, Mukai K and Tachibana M: Annexin II represents metastatic potential in clear-cell renal cell carcinoma. Br J Cancer 101: 287-294, 2009.

23. Zhang W, Zhao P, Xu XL, Cai L, Song ZS, Cao DY, Tao KS, Zhou WP, Chen ZN and Dou KF: Annexin A2 promotes the migration and invasion of human hepatocellular carcinoma cells in vitro by regulating the shedding of CD147-harboring microvesicles from tumor cells. PLoS One 8: e67268, 2013.

24. Oh M, Rhee S, Moon JH, Chae H, Lee S, Kang J and Kim S: Literature-based condition-specific miRNA-mRNA target prediction. PLoS One 12: e0174999, 2017.

25. Dweep H, Sticht C, Pandey P and Gretz N: miRWalk-database: Prediction of possible miRNA binding sites by 'walking' the genes of three genomes. J Biomed Inform 44: 839-847, 2011

26. Xiao H, Xiao W, Cao J, Li H, Guan W, Guo X, Chen K, Zheng T, Ye Z, Wang J and Xu H: miR-206 functions as a novel cell cycle regulator and tumor suppressor in clear-cell renal cell carcinoma. Cancer Lett 374: 107-116, 2016.

27. Ren XL, He GY, Li XM, Men H, Yi LZ, Lu GF, Xin SN, Wu PX, Li YL, Liao WT, et al: MicroRNA-206 functions as a tumor suppressor in colorectal cancer by targeting FMNL2. J Cancer Res Clin Oncol 142: 581-592, 2016.

28. Keklikoglou I, Hosaka K, Bender C, Bott A, Koerner C, Mitra D, Will R, Woerner A, Muenstermann E, Wilhelm $\mathrm{H}$, et al: MicroRNA-206 functions as a pleiotropic modulator of cell proliferation, invasion and lymphangiogenesis in pancreatic adenocarcinoma by targeting ANXA2 and KRAS genes. Oncogene 34: 4867-4878, 2015.

29. Chen L, Li YS, Cui J, Ning JN, Wang GS, Qian GS, Lu KZ and Yi B: MiR-206 controls the phenotypic modulation of pulmonary arterial smooth muscle cells induced by serum from rats with hepatopulmonary syndrome by regulating the target gene, annexin A2. Cell Physiol Biochem 34: 1768-1779, 2014
30. Scanlon CS, Van Tubergen EA, Inglehart RC and D'Silva NJ: Biomarkers of epithelial-mesenchymal transition in squamous cell carcinoma. J Dent Res 92: 114-121, 2013.

31. Martínez-Ramírez AS, Garay E, García-Carrancá A and Vázquez-Cuevas FG: The P2RY2 receptor induces carcinoma cell migration and EMT through cross-talk with epidermal growth factor receptor. J Cell Biochem 117: 1016-1026, 2016.

32. Carvalho-Cruz P, Alisson-Silva F, Todeschini AR and Dias WB: Cellular glycosylation senses metabolic changes and modulates cell plasticity during epithelial to mesenchymal transition. Dev Dyn 247: 481-491, 2018

33. Kaushik NK, Kaushik N, Yoo KC, Uddin N, Kim JS, Lee SJ and Choi EH: Low doses of PEG-coated gold nanoparticles sensitize solid tumors to cold plasma by blocking the PI3K/AKT-driven signaling axis to suppress cellular transformation by inhibiting growth and EMT. Biomaterials 87: 118-130, 2016.

34. Yu AQ, Ding Y, Li CL, Yang Y, Yan SR and Li DS: TALEN-induced disruption of Nanog expression results in reduced proliferation, invasiveness and migration, increased chemosensitivity and reversal of EMT in HepG2 cells. Oncol Rep 35: 1657-1663, 2016

35. Chen HN, Yuan K, Xie N, Wang K, Huang Z, Chen Y, Dou Q, Wu M, Nice EC, Zhou ZG and Huang C: PDLIM1 Stabilizes the E-Cadherin/ $\beta$-Catenin complex to prevent epithelial-mesenchymal transition and metastatic potential of colorectal cancer cells. Cancer Res 76: 1122-1134, 2016.

This work is licensed under a Creative Commons Attribution-NonCommercial-NoDerivatives 4.0 International (CC BY-NC-ND 4.0) License. 